\title{
Valoração e percepção ambiental: estudo de caso no baixo curso do rio Cocó, Fortaleza, Ceará, Brasil
}

O rio Cocó possui um dos principais recursos hídricos da Região Metropolitana de Fortaleza e contendo múltiplos usos ao longo do seu curso e manguezal. Nesse sentido, o presente estudo teve como objetivos estimar o valor econômico ambiental de um trecho do rio localizado em seu baixo curso e verificar a percepção ambiental dos moradores e frequentadores do local. A DAP média encontrada foi de R\$ 1,93 per capita por mês e o valor médio mensal de $\mathrm{R} \$ \mathbf{8 2 . 1 7 5 , 5 4}$ considerando a população total dos entornos da área estudada. De acordo com a percepção ambiental dos entrevistados, o bem-estar e o lazer são os principais benefícios do trecho à Fortaleza, além disso, 79\% não consome o pescado da região atribuído ao aspecto visual do rio e poluição do mesmo.

Palavras-chave: Valoração Ambiental; Percepção; Economia Verde.

\section{Environmental valuation and perception: a case study in the lower course of the Cocó river, Fortaleza, Ceará, Brazil}

\begin{abstract}
The River Cocó has a main water resources in the metropolitan area of Fortaleza and containing multiple uses throughout its course and mangroves. In this sense, the present study aimed to estimate the environmental economic value of a stretch of river located in its lower course and check the environmental awareness of residents and regulars of the place. The average DAP found was R\$ 1.93 per capita per month and the average monthly amount of $R \$ 82,175.54$ considering the total population of the surroundings of the study area. According to the environmental perception of respondents, the well-being and pleasure are the main benefits of the stretch to Fortaleza, in addition, $79 \%$ did not consume the fish of the region assigned to the visual appearance of the river and pollution of it.

Keywords: Environmental Valuation; Perception; Green Economy.
\end{abstract}

Topic: Valoração e Economia Ambiental

Reviewed anonymously in the process of blind peer.
Received: 10/10/2016

Approved: 05/01/2017
Beatriz França Machado Alves de Almeida Universidade Federal do Ceará, Brasil. http://lattes.cnpq.br/6905149172831482 beatrizfmaa@hotmail.com

Kamila Vieira de Mendonça

Universidade Federal do Ceará, Brasil. http://lattes.cnpq.br/8988759234165935

kamilavm@gmail.com

Fábio de Oliveira Matos

Universidade Federal do Ceará, Brasil.

http://lattes.cnpq.br/0103822859471277

fabiomoria@yahoo.com.br
Referencing this:

ALMEIDA, B. F. M. A.; MENDONÇA, K. V.; MATOS, F. O.. Valoração e percepção ambiental: estudo de caso no baixo curso do rio Cocó, Fortaleza, Ceará, Brasil. Revista Ibero-Americana de Ciências Ambientais, v.8, n.2, p.299-306, 2017. DOI: http://doi.org/10.6008/SPC2179-6858.2017.002.0024 


\section{INTRODUÇÃO}

As principais problemáticas enfrentadas atualmente não devem ser analisadas e estudadas isoladamente, pois elas possuem causas e consequências sistêmicas, resultados de uma teia interligada de agentes e processos (CAPRA, 2006). Corroborando com a teoria de Capra et al. (2005) defende uma maior aproximação entre as ciências, para o autor os estudos físico-químicos do ambiente não dialogam com os contextos sociais e políticos.

$\mathrm{Na}$ Economia, a busca por pesquisas mais holísticas culminou em uma ramificação, denominada de Economia Verde que possui dentre seus artifícios a Valoração Econômica Ambiental (VEA) (MAY et al., 2010). Em seu livro, Motta (1998) afirma que o método de VEA estima um valor monetário de um recurso ambiental em relação aos outros bens e serviços existentes na economia. Tal valor pode ser utilizado como um indicador de priorização uma vez que, a ausência de preços para os recursos ambientais e, consequentemente, os serviços por eles prestados trazem uma problemática: uso excessivo dos recursos (NOGUEIRA et al., 2000).

Atualmente, a busca por um modelo mais sustentável de uso dos recursos naturais e gestão de cidades que possa solucionar as problemáticas e os impactos atuais e futuros requer estudos mais integrados e contextualizados como mencionados anteriormente. Nesse contexto, a VEA juntamente com a Percepção Ambiental integra-se como componentes metodológicos que possam fomentar as escolhas e usos de instrumentos para uma melhor gestão urbana.

Dessa forma, o presente estudo tem como objetivo geral estimar o valor ambiental econômico ambiental de um trecho do rio Cocó. Adicionalmente, verificar a percepção ambiental, obter e avaliar dados socioeconômicos dos residentes dos entornos do trecho do rio.

\section{METODOLOGIA}

\section{Área de Estudo}

O rio Cocó constitui um dos principais recursos hídricos da Região Metropolitana de Fortaleza (RMF) e configura-se como um rio urbano. Sua nascente fica situada na Serra de Aratanha, no município de Pacatuba. A bacia do rio Cocó compreende as áreas dos municípios de Aquiraz, Maranguape, Pacatuba e Fortaleza e possui uma área de aproximadamente $485 \mathrm{~km}^{2}$, com um comprimento total do rio principal de cerca de 50 km (FORTALEZA, 2003; LOUREIRO, 2005; SEMACE, 2005).

O rio Cocó serve como meio de sustento para comunidades que vivem da pesca ao longo do curso e em seu manguezal ele assume um papel de significativa importância para a qualidade de vida local, devido a fatores ligados ao microclima, equilíbrio ecológico, bem como do ponto de vista paisagístico, dispondo de potencial ecoturístico e desempenhando um papel de lazer para os cidadãos; seu curso recebe um barramento no qual resulta o reservatório Gavião, fundamental para o abastecimento da Região Metropolitana de Fortaleza (COGERH, 2002). 
O baixo curso do rio Cocó é conferido quando seu leito se localiza próximo ao bairro Castelão em Fortaleza e segue até sua foz na praia da Sabiaguaba e é onde se encontra o setor mais urbanizado da bacia (ZANELLA et $a l, 2013$ ).

Ao longo do seu curso, o rio Cocó recebe vários impactos ambientais, tais como o lançamento de efluentes de esgotamentos sanitários domésticos sem tratamentos das residências instaladas em suas margens e próximas a ele; supressão da mata ciliar e vegetação de entorno; infiltração do solo de suas margens pelo avanço da urbanização e recebimento de chorume do antigo aterro sanitário do Jangurussu que mesmo após sua desativação continua sendo fonte de cargas contaminantes ao rio (ZANELLA et al., 2013).

Além dos impactos ambientais, o rio possui um histórico de lutas e mobilizações socioambientais, sendo as mais significativas o movimento surgido em 1978 pela defesa das áreas de mangue e a criação do Parque Ecológico do Cocó (CARDOSO et al., 2009) e mais recentemente, em 2014, uma ocupação social de parte do manguezal organizada por militantes ambientalistas em entrave contra a construção de um viaduto e em prol da legalização do parque.

As manifestações ocorridas em 2014 trouxeram o debate para a sociedade civil em relação à gestão urbana, preservação de áreas verdes, qualidade ambiental do rio e mobilidade urbana. Nesse contexto, a determinação do valor econômico ambiental e o conhecimento das percepções ambientais das populações do entorno do rio, é imprescindível para as tomadas de decisões, soluções dos conflitos e melhoria da qualidade ambiental do recurso.

\section{Amostragem}

A área de amostragem detém o perfil mais urbanizado em comparação às outras que margeiam o rio ao longo do seu baixo curso. $\mathrm{O}$ trecho escolhido faz parte da regional $\mathrm{VI}^{1}$ de Fortaleza. Para valorar o trecho foram aplicados questionários semiestruturados nos entornos dos bairros que margeiam o ponto amostral escolhido. Os bairros Aerolândia (E 1) que possui uma população de 11.824 habitantes e o bairro Jardim das Oliveiras (E 2) que tem uma população de 30.754 habitantes. (PREFEITURA DE FORTALEZA, 2014)

Para a pesquisa foi utilizado o Método de Valoração Contingente e a categoria Disposição a Pagar (DAP) que é estimada por meio de uma função de distribuição das respostas afirmativas e correlacionadas com uma função de utilidade indireta. Por meio da média ou mediana das DAP's multiplicada pelo total da população, tem-se o valor econômico total do recurso ambiental (MOTTA, 2006).

Segundo Hanemann et al. (2012), o método de valoração contingente busca mensurar monetariamente a variação no nível de bem-estar dos indivíduos devido a uma alteração na provisão de um bem por meio da função utilidade indireta do entrevistado através da função:

$$
\mathrm{U}=\mathrm{V}(\mathrm{J}, \mathrm{Y}, \mathrm{S})(01)
$$

\footnotetext{
${ }^{1}$ Conjunto de bairros divididos geograficamente e que possuem uma Secretaria que permitem uma melhor gestão urbana de Fortaleza. 
Onde U é a utilidade; J é a variável binária, em que J = 1 se o indivíduo aceita a DAP o que significa acesso ao bem público e 0 se não aceita, ou seja, sem acesso; $Y$ é a renda do indivíduo; e, $\mathrm{S}$ são as características socioeconômicas do entrevistado. A partir dessa função utilidade o modelo logit é estimado pelo método de máxima verossimilhança pela seguinte forma funcional:

$$
\ln \left[\frac{\operatorname{Pr}_{i}(\operatorname{sim})}{\operatorname{Pr}_{i}(\text { não })}\right]=\ln \left[\frac{\operatorname{Pr}_{i}(\operatorname{sim})}{1-\operatorname{Pr}_{i}(\operatorname{sim})}\right]=X_{i}^{\prime} \beta=\beta_{0}+\sum \beta_{j} X_{i j}+\varepsilon_{i}
$$

A DAP é dada pela mediana no ponto em que $\operatorname{Pr}(\operatorname{Sim})=\operatorname{Pr}(\mathrm{Não})=0,5$. Assim:

$$
\bar{X}=\int_{0}^{\infty}[1-G(X)] d x-\int_{-\infty}^{0}[G(X)] d x
$$

Onde se obtém:

$$
\bar{X}=-\frac{\hat{\beta}^{*}}{\hat{\beta_{1}}}
$$

Em que o denominador é a estimativa do parâmetro do valor sugerido aos entrevistados e o numerador é o valor resultante da soma da constante com o produto de todos os outros coeficientes da estimativa, multiplicados pelas respectivas médias.

No estudo, foram aplicados questionários pilotos em fevereiro de 2014 com 30 indivíduos, de modo a testar a confiabilidade das questões. Depois de revistos, determinou-se o tamanho da amostra com número mínimo de 96 elementos. Esse valor foi obtido considerando-se um erro amostral de 10\% com nível de significância de $95 \%$. Os questionários possuem formação de um conjunto de valores possíveis que podem representar a DAP. O entrevistador escolhe, aleatoriamente, um valor e apresenta ao entrevistado que vai responder se está ou não disposto a pagar. Com isso, obtemos um conjunto de respostas binárias representadas por 1 ou 0.0 formato de referendo é bastante utilizado por apresentar menor quantidade de vieses por parte dos entrevistados e se assemelham às condições reais de mercado.

O questionário definitivo foi estruturado com quatorze questões que objetivaram a coleta de variáveis socioeconômicas e ambientais dos indivíduos amostrados, bem como de opiniões pessoais sobre a disposição a pagar. A aplicação dos questionários definitivos ocorreu entre os meses de agosto de 2014 e fevereiro de 2015 em diversos horários e dias da semana, no período da manhã e da tarde.

No questionário perguntas acerca da utilização do trecho do rio e importância do rio à Fortaleza compuseram a componente de percepção ambiental além de anotações no diário de campo das opiniões e comentários dos entrevistados.

\section{RESULTADOS E DISCUSSÃO}

Considerando o universo amostral total $(n=96)$, foram aplicados 48 questionários no Jardim das Oliveiras e 48 na Aerolândia. No Jardim das Oliveiras a média de idade é de 45 anos, a renda mensal líquida 
média é de R\$ 3.550,00 e o gênero predominante foi o masculino; na Aerolândia, a idade média é de 47 anos, a renda média é de $\mathrm{R} \$ 1.990,00$ e, também, a maioria dos entrevistados foi do gênero masculino.

Quanto ao grau de instrução, no Jardim das Oliveiras e na Aerolândia a predominância é de ensino superior incompleto. Em relação à atividade de fonte de renda, as três atividades mais citadas no Jardim das Oliveiras foram trabalho formal $(n=13)$, trabalho autônomo $(n=11)$ e estudantes $(n=10)$; na Aerolândia, os resultados foram trabalho formal $(n=15)$, trabalho autônomo $(n=8)$ e aposentadoria $(n=7)$ e dono(a) de casa $(n=7)$.

A atividade de pesca é comum no local, por conta disso, os frequentadores foram questionados a respeito do consumo de pescado. Considerando os dois entornos, 20 pessoas afirmaram consumir o peixe do trecho analisado. Os entrevistados que não consomem peixe atribuíram à aparência poluída do rio como motivo principal. As frequências de consumo do pescado local apresentadas foram de 1-2 vezes por semana $(n=17)$ e 3-4 vezes por semana $(n=3)$.

Quanto à percepção ambiental dos indivíduos que vivem nos entornos do rio, foram apresentadas problemáticas ambientais como poluição da água, mau cheiro e danificação da infraestrutura (calçada, bancos, lixeiras etc.) pelos próprios moradores, conforme são apresentados na transcrição dos relatos R1, $R 2, R 3$, respectivamente:

O rio é muito bom, mas está muito poluído, tem muito aguapé que traz muito mosquito para dentro de casa.'

As pessoas vêm e ajeitam a calçada, colocam os bancos, fica tudo bonito, ótimo para caminhar porque o vento aqui é bom. Mas, com menos de três meses, já está tudo quebrado, o pessoal aqui não cuida do que tem.'

“Antigamente aqui tinha muito mais peixe. Mas, agora tá tudo poluído. Só tem os peixes de lama mesmo.' (R1, R2, R3, 2014)

Sobre quais os benefícios proporcionados pelo rio à cidade, as respostas foram similares em relação aos dois bairros (Gráfico 1). Resultados semelhantes foram apresentados por Passareli (2013) em estudo feito sobre a percepção e a valoração econômica ambiental do manguezal na Paraíba do Sul, Rio de Janeiro. No estudo, o benefício de lazer também foi apresentado como um dos citados junto com qualidade ambiental e preservação da biodiversidade.

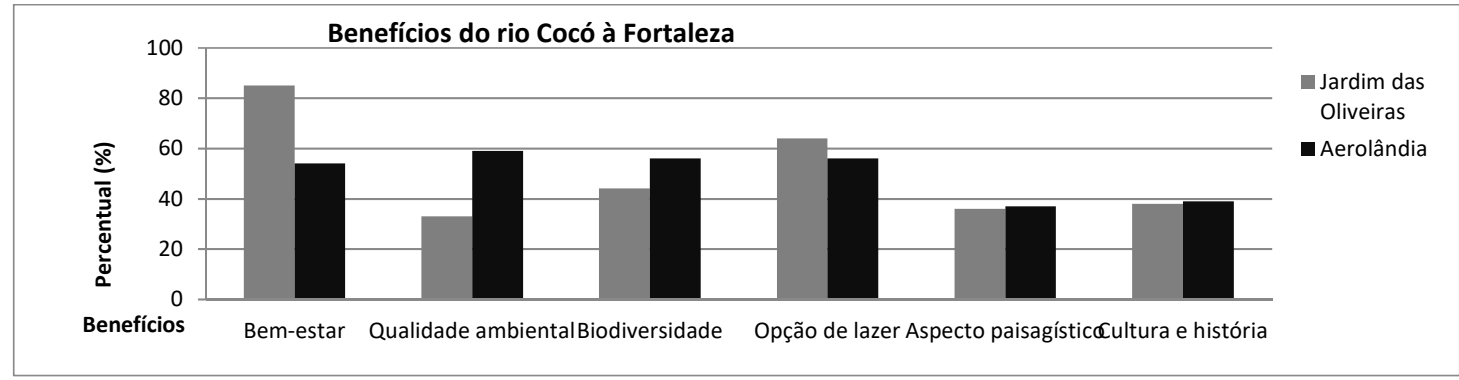

Gráfico 01: Benefícios do rio Cocó à Fortaleza.

No Jardim das Oliveiras, o bem-estar ( $85 \%$ ) e a opção de lazer (64\%) se destacam como os benefícios mais citados; na Aerolândia há uma menor discrepância entre os benefícios, sendo o bem-estar (54\%), qualidade ambiental (59\%), biodiversidade (56\%) e opção de lazer (56\%) como as opções apresentadas. 
Esses resultados podem estar relacionados às formas de utilização do rio. No Jardim das Oliveiras, o lazer $(n=9)$ e a passagem $(n=14)$ foram as mais relatadas, porém a não utilização do rio $(n=14)$ também foi apresentada em um nível alto. Quanto ao lazer e a passagem foram mencionadas caminhadas e caminhos pelas margens para ir a lugares como mercearias, escola ou casa de amigos e/ou familiares. A não utilização do rio foi apontada por motivos de carência e depredação da infraestrutura e insegurança, principalmente no período da noite; na Aerolândia, o lazer $(n=16)$ e a passagem $(n=19)$ apresentam a maioria maior frequência nessa categoria. Foram mencionadas caminhadas e jogos de futebol como atividades de lazer e passagem por caminhos para deslocamentos etc.

Os relatos como instrumento de percepção ambiental e que podem elucidar resultados quantitativos podem ser importantes em uma gestão urbana mais participativa como apresentado por Silva (2014) em estudo com pescadores e proprietários comerciais do rio Paraguai, no estado do Mato Grosso. Nesse estudo, há uma análise geral acerca da percepção ambiental, por meio do conhecimento dela para que possa haver uma avaliação mais holística em relação à valoração econômica ambiental.

Quanto ao valor econômico ambiental, os resultados mostram que $34 \%(n=33)$ dos entrevistados estão dispostos a pagar um valor para manutenção e proteção do trecho do rio. Assim, a DAP média encontrada para os indivíduos que se dispuseram a pagar foi de $\mathrm{R} \$ 1,93$ per capita por mês. Ao calcularmos esse valor para a população dos dois entornos temos que o valor médio é de $\mathrm{R} \$ 82.175,54$ por mês.

Hildebrand et al. (2002) encontrou um valor semelhante ao presente estudo apresentando uma DAP média de $\mathrm{R} \$ 1$ 1,21/mês e $\mathrm{R} \$ 29 ., 040 /$ mês para um bosque em Curitiba. Porém, Silva et al. (2004) apresentaram uma DAP média de R\$ 7,60/mês do parque Chico Mendes em Rio Branco. Em estudo feito em Salvador com um parque urbano, Souza \& Mota (2006), encontraram uma DAP média de R\$\$7,72/mês resultados superiores ao presente estudo.

As justificativas apresentadas por $66 \%(n=63)$ dos entrevistados que não se dispuseram a pagar foram: os impostos pagos deveriam cobrir tais gastos e a "taxa" deveria existir para todos os moradores de Fortaleza, e não, somente a quem reside nas proximidades do trecho analisado. Justificativas semelhantes foram encontradas por Hildebrand et al. (2002) e João et al. (2008), onde, neste último. No estudo de João et al. (2008) realizado em um Engenho - de SP, foi encontrado um resultado nulo para a DAP.

A poluição do rio e depredação da infraestrutura como já foi discutido no item anteriormente, podem ser fatores que influenciam os indivíduos a optarem por um valor nulo ou baixo para o trecho analisado. 0 que corrobora com as variáveis explicativas provenientes da estimação do modelo Logit apresentadas na (Tabela 1). A frequência de utilização tem um sinal negativo indicando que a variável tem um efeito negativo na disposição a pagar. Esse resultado sugere que a frequência de utilização tem um efeito negativo na probabilidade de pagamento, isso se dá pois, ao frequentarem trecho do rio, os indivíduos conhecem suas problemáticas, como já citadas, a poluição e a depredação da infraestrutura. A baixa frequência dos que se dispuseram a pagar também é sugerida como explicação para o valor menor em relação a outros estudos citados anteriormente. 
Tabela 1: Estimativa dos parâmetros e efeito marginal da função de disposição a pagar pela manutenção/preservação do trecho do rio Cocó, Fortaleza.

\begin{tabular}{|c|c|c|}
\hline Variáveis explicativas & Coeficientes de regressão & Efeito Marginal \\
\hline Constante & $1,636^{*}$ & \\
\hline Frequência Utilização & $-0.377^{*}$ & -0.076 \\
\hline Valor da DAP & $-0,149 *$ & $-0,030$ \\
\hline $\begin{array}{l}\text { Valor da Estatistica Qui- } \\
\text { Quadrado }\end{array}$ & 10,66 & \\
\hline Valor-p & 0,0049 & \\
\hline Pseudo $\mathrm{R}^{2}$ & 0,0863 & \\
\hline
\end{tabular}

Mesmo apresentando problemáticas socioambientais, bem como, poluição da água e carência na infraestrutura, os indivíduos reconhecem e percebem a importância do rio e seus benefícios à cidade. De acordo Proshanky (1978) e Bomfim (2010) a promoção da apropriação e utilização de espaços públicos contribui com uma gestão eficiente em que promovem a qualidade de vida aos cidadãos e ao mesmo tempo uma preservação do meio ambiente. O reconhecimento, bem como, a valoração desses dessas áreas são potencializadores para uma gestão integralizada e sustentável das cidades.

\section{CONCLUSÃO}

Os resultados obtidos apontam que as relações dos indivíduos com o rio se dá, principalmente, por fatores de qualidade de vida (bem-estar, $57 \%$ e opção de lazer, $50 \%$ ) e que a poluição do rio e a degradação da infraestrutura dos arredores (calçada, lixeira etc.) também foi um fator considerável para o valor ambiental atribuído pelos entrevistados ao trecho estudado.

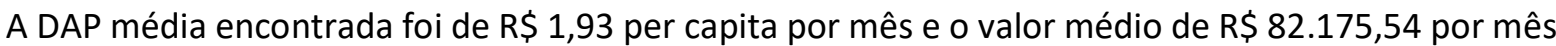
considerando a população total dos entornos. Esses valores podem auxiliar na gestão pública da cidade em que é considerável o valor encontrado ao rio para as populações do entorno. O que sugere, portanto, que haja um esforço coletivo (político e social) para uma reaproximação entre a população e o rio Cocó, na qual, haja a diminuição das problemáticas apresentadas.

É percebido um círculo vicioso em que quanto mais o rio encontra-se degradado mais as pessoas se afastam e se desapropriam do mesmo, e quanto mais há o afastamento e desapropriação, processos de reversão e melhoras da qualidade do rio diminuem. A apropriação do espaço pode ser um mecanismo catalisador da participação da sociedade nos processos de discussão e de tomada de decisão relacionadas com as questões e políticas públicas, ou seja, nos processos decisórios quanto à qualidade ambiental e saúde pública. Os resultados apresentados mostram que o rio Cocó fornece benefícios e que eles são reconhecidos pela população. O que pode auxiliar na gestão pública municipal.

\section{REFERÊNCIAS}

BOMFIM, Z. A. C.. Cidade e afetividade: estima e construção dos mapas afetivos de Bercelona e de São Paulo. São Paulo: UFC, 2010.

CAPRA, F.. A teia da vida: uma nova compreensão científica dos sistemas vivos. Cultrix, 2006
CARDOSO, A. M. F.; MATOS, K. S. A. L.. O movimento ambientalista e as perspectivas ambientais em grupos populares de Fortaleza. Educação Ambiental e Sustentabilidade, p.15-29, 2009.

FORTALEZA. Inventário ambiental dos sistemas hídricos e orla marítima do município de Fortaleza: Relatório de andamento. Fortaleza: PMF, 2003. 
FORTALEZA. Site da cidade de Fortaleza com informações gerais, censos e regionais. 2015.

HILDEBRAND, E.; GRAÇA, L. R.; HOEFLISH, V. A.. Valoração contingente na avaliação econômica de áreas verdes urbanas. Revista Floresta, v.32, n.1, 2002.

LOUREIRO J. M. B.. Parque Ecológico do Cocó: A produção do espaço urbano no entorno de áreas de proteção ambiental. Revista Mercartor, v.4, n.8, 2005.

MAY, P. H.. Economia do Meio Ambiente: teoria e prática. 2 ed. Elsevier, 2010.

MOTTA, R. S.. Manual para valoração econômica de recursos ambientais. Rio de Janeiro, 1998.

MOTTA, R. S.. Economia Ambiental. Rio de Janeiro: FGV, 2006.
ROHDE, G. M.. Epistemologia Ambiental. Porto Alegre: EDIPUCRS, 2005.

SILVA, E. V.; SOUZA, M. M. A.. Principais formas de uso e ocupação dos manguezais do Estado do Ceará. Caderno de Cultura e Ciência, v.1, n.1, 2005.

SILVA, R. V.. Os olhares dos pescadores profissionais e proprietários comerciais, sobre o rio Paraguai em Cárceres, Mato Grosso. Revista Brasileira de Ciências Ambientais, n.32, 2014.

SOUZA, G. B.; MOTA, J. A.. Valoração econômica ambiental de áreas de recreação: o caso do Parque Metropolitano do Pituaçu, Salvador, BA. Revista de Economia, v.32, n.1, 2006.

ZANELLA, M. E.; OLIMPIO, J. L.; COSTA, M. C. L.; DANTAS, E. W. C.. Vulnerabilidade socioambiental do baixo curso da bacia hidrográfica do rio Cocó, Fortaleza - CE. Revista Sociedade e Natureza, v.25, n.2, p.317-322, 2013. 